Article

\title{
Optimization Design of an Inductive Energy Harvesting Device for Wireless Power Supply System Overhead High-Voltage Power Lines
}

\author{
Wei Wang, Xueliang Huang *, Linlin Tan, Jinpeng Guo and Han Liu
}

Department of Electrical Engineering, Southeast University, No. 2 Sipailou, Nanjing 210096, Jiangsu, China; wangw_seu@163.com (W.W.); linsky_432@163.com (L.T.); gjp1992@sina.com (J.G.); liuhan199305@163.com (H.L.)

* Correspondence: xlhuang@seu.edu.cn; Tel.: +86-25-8379-4691; Fax: +86-25-8379-1696

Academic Editor: Ling Bing Kong

Received: 23 December 2015; Accepted: 21 March 2016; Published: 26 March 2016

\begin{abstract}
Overhead high voltage power line (HVPL) online monitoring equipment is playing an increasingly important role in smart grids, but the power supply is an obstacle to such systems' stable and safe operation, so in this work a hybrid wireless power supply system, integrated with inductive energy harvesting and wireless power transmitting, is proposed. The energy harvesting device extracts energy from the HVPL and transfers that from the power line to monitoring equipment on transmission towers by transmitting and receiving coils, which are in a magnetically coupled resonant configuration. In this paper, the optimization design of online energy harvesting devices is analyzed emphatically by taking both HVPL insulation distance and wireless power supply efficiency into account. It is found that essential parameters contributing to more extracted energy include large core inner radius, core radial thickness, core height and small core gap within the threshold constraints. In addition, there is an optimal secondary coil turn that can maximize extracted energy when other parameters remain fixed. A simple and flexible control strategy is then introduced to limit power fluctuations caused by current variations. The optimization methods are finally verified experimentally.
\end{abstract}

Keywords: high voltage power line; wireless power supply; energy harvesting; power fluctuation

\section{Introduction}

Industrial electricity demand has been drastically increasing with the spread and intensification of industrial development. As a result, high voltage power lines (HVPLs) are playing an increasingly important role in the connection between the western and eastern regions of China to achieve the economic coordination. However, damage to HVPLs caused by strong winds, extreme climate, icing, lightning, contamination, tower tilting, etc. are inevitable due to the terrible working conditions in wild and remote areas as well as relatively long distances between towers [1,2]. In order to avoid possible paralysis of the power grid, real-time online monitoring is applied to supervise the potential dangers to HVPLs with the help of real-time status monitoring equipment.

In recent years, domestic and foreign scholars have proposed several methods for HVPL fault diagnosis and online monitoring [3], but the problem of an convenient and efficient power supply for monitoring equipment remains unresolved. Previous studies [4-7] have introduced several commonly-used power supply methods: (1) on the basis of the capacitor dividing principle, energy obtained from electric fields around the power line can be used to power the monitoring equipment; (2) lasers beams carrying high energy are sent from the low voltage side to the receiving device located in the high voltage side and then transformed into electricity; (3) a new way to supply power is to comprehensively utilize photovoltaic, wind and battery energy, which is the most mature one and 
has already been installed on some power lines. Nevertheless, the solutions mentioned above share some common problems such as being subject to the influences from the harsh external environment, low efficiency of photoelectric conversion devices, small receiving energy, short battery life and so on. Previous studies [8-11] presented a novel online energy harvesting device based on electromagnetic induction. Electricity is acquired by a current transformer (CT) directly attached to the high voltage power lines. This approach shows high stability, high efficiency and convenience of power supply with equal potential, particularly when it comes to monitoring equipment, such as inspection robots, which are directly attached to the line. However, disadvantages, such as low extracted power, poor saturation features, voltage distortion due to increased current and over-heating are also prominent. Based on the consideration of core saturation, a US patent [12] proposed a new type of separation core, but unfortunately only the structure and method of installation are mentioned. In addition, other studies also found that $\mathrm{Mn}-\mathrm{Zn}$ ferrite and other materials with higher permeability can greatly reduce the eddy current losses and improve the core power density $[13,14]$. However, accurate analysis and design of inductive energy harvesting devices, which can achieve high-level power and avoid distortion of voltage, are rarely reported. Even though inductive energy harvesting can resolve the problem of powering monitoring devices on HVPLs which have the same potential as line voltage, the way of power transmission used to connect the HVPL and monitoring equipment on the transmission tower should be carefully chosen due to the extremely large potential difference. In sum, those power supply methods share a common problem, that is, power transmission from HVPLs to monitoring equipment on the transmission tower is tough to accomplish. Accordingly, [15] proposed the use of optical fiber to resolve the electrical insulation problem. It is undeniable that optical fiber has its own unique advantages in dealing with electrical insulation, but harsh environmental conditions like strong winds and blizzards will make the fiber become much more fragile, so the use of fiber optics is not a permanent solution.

To overcome the above problems and improve the stability and security of the power supply of monitoring equipment, a new power supply mode to achieve energy harvesting by combining electromagnetic induction and wireless power transmitting technology is proposed. According to [16], in 2007 Kurs at the Massachusetts Institute of Technology (MIT) successfully lit a $60 \mathrm{~W}$ bulb over a distance in excess of $2 \mathrm{~m}$ by using magnetically coupling resonant wireless power transmission. With reference to "Electric Power Industry Standard of the People's Republic of China-The Technical Code for Designing 110-500 kV Overhead Transmission Line", seven or eight suspension insulator strings are needed for a $110 \mathrm{kV}$ overhead line which covers about an insulation distance of $1.1 \mathrm{~m}$, and this paper thus takes the $110 \mathrm{kV}$ HVPL as an example. As far as we are concerned, there is nothing relevant to the application of magnetically coupling resonant wireless power transmitting technology in this field. Therefore, the magnetically coupling resonant wireless power transmitting technology provides a new solution to power $110 \mathrm{kV}$ HVPL on-line monitoring equipment.

In order to solve the problem of powering online monitoring equipment, a combination of inductive energy harvesting and magnetically coupling resonant wireless power transfer is proposed. The power demand of wireless power transfer devices as well as the distortion of output power and overheating should be taken into consideration. As a result, a particular and precise design relevant to enhance output power and avoid the chances of voltage distortion is necessary. There are some inductive energy harvesting device prototypes, but most cannot provide enough power and have current fluctuation problems. One cause accounting for this is that detailed design methods of inductive energy harvesting devices are rarely introduced. Instead, the design experience of traditional CT for measurements is used as a reference and consequently, there are still many practical application problems.

This paper mainly focuses on $110 \mathrm{kV}$ high voltage lines and aims to develop an energy harvesting device by electromagnetic induction and wireless power transmission technology. The energy harvesting device not only should meet the power needs of the wireless power transmission system, but also needs to adapt to the normal current fluctuations of high voltage power lines. The factors 
which may influence the working features of the energy harvesting device are further analyzed. Optimization design of specific parameters is also taken into consideration with computational and experimental verification. The work we do should be helpful to promote the practical implementation of HVPL wireless power supply systems. Most importantly, it also provides a new solution to the supply problem of monitoring equipment located on transmission towers, such as bird driving devices, data acquisition units, signal transceiver repeaters, temperature monitoring sensors, insulator contamination testing sensors, monitoring cameras and so on.

\section{System Modeling and Analysis of the Wireless Power Supply System}

The HVPL wireless power supply system consists of a energy harvesting device based on the electromagnetic induction principal and a wireless power transmission system. Electricity is firstly obtained from the HVPL by electromagnetic induction [17] and then converted into a high frequency signal by a high frequency conversion device. Secondly, the transmitter in the wireless power transmission system powered by the high frequency signal transmits the energy to a receiver located beyond the insulation distance through magnetically coupling resonant wireless power transmission. Finally, the received energy is rectified and voltage-stabilized to power the online monitoring equipment. Specific devices used are shown as follows: energy harvesting device attached to the HVPL, protection control, energy storage and discharge circuit to relieve the effect of current fluctuations, high frequency conversion device to convert the power frequency into high frequency, energy receiver located on the transmission tower to receive electromagnetic energy and transform it into high frequency electricity, rectification and voltage regulator circuit to transform high frequency electricity into direct current (DC) electric energy for powering online monitoring devices. Among them, the power transmission and receiving coils are connected with tuning capacitors in series, which makes the wireless power transmitting system be a state of series resonance. The overall system configuration is shown in Figure 1.

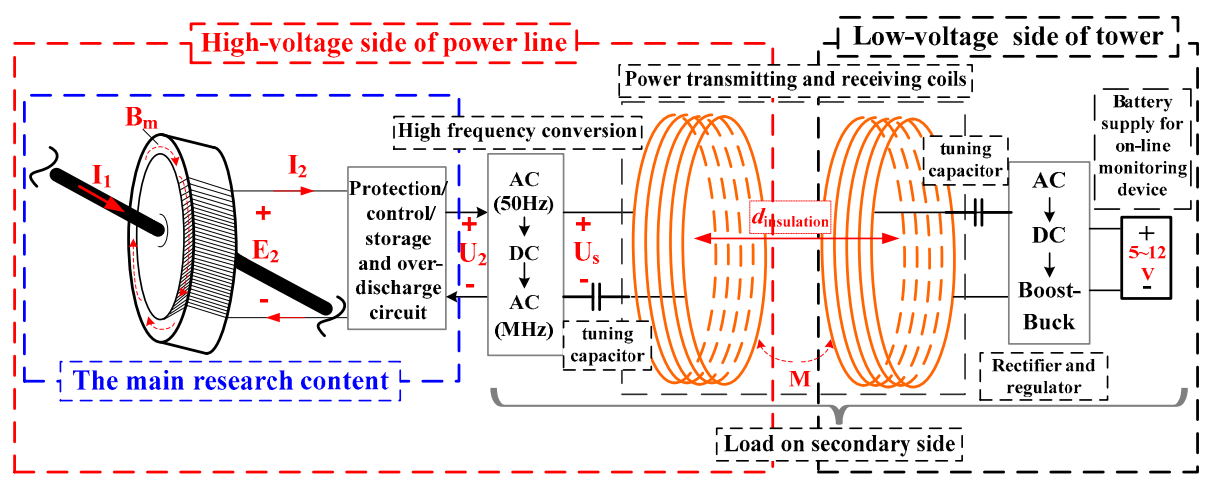

Figure 1. Structure of a wireless power supply system for high voltage power lines (HVPLs). AC, alternating current; DC, direct current.

The voltage of HVPL monitoring equipment on the market is generally DC 5-2 V while the power rating ranges from $1 \mathrm{~W}$ to $10 \mathrm{~W}$. For a typical wireless power transmitting system, $\mathrm{L}_{t}$ and $\mathrm{L}_{\mathrm{r}}$ denote the inductance of the transmitting coil and receiving coil, respectively. $\mathrm{I}_{t}$ and $\mathrm{I}_{\mathrm{r}}$ accordingly denote the current on the transmission and receiving sides. It has been proved that the output power is closely related to the working frequency, and specifically that a higher frequency can transfer more energy within a long distance $[18,19]$. With reference to "Electric Power Industry Standard of the People's Republic of China-The Technical Code for Designing 110-500 kV Overhead Transmission Line", the insulation distance of $110 \mathrm{kV}$ HVPL is approximate $1.1 \mathrm{~m}$, so the magnitude of the working frequency should be set around MHz. In this paper, the transmission coil and the receiving coil are connected in series with the tuning capacitor. The Kirchhoff's voltage law (KVL) equation can de expressed by: 


$$
\left\{\begin{array}{c}
\dot{I}_{t} Z_{1}-j \omega M \dot{I}_{r}=\dot{U}_{s} \\
\dot{I}_{r} Z_{2}-j \omega M \dot{I}_{t}=0
\end{array}\right.
$$

The transmission and receiving current can be calculated and written as:

$$
\left\{\begin{array}{l}
\dot{I}_{t}=\frac{Z_{2} \dot{U}_{s}}{Z_{1} Z_{2}+(\omega M)^{2}} \\
\dot{I}_{r}=\frac{j \omega M \dot{U}_{s}}{Z_{1} Z_{2}+(\omega M)^{2}}
\end{array}\right.
$$

Here $Z_{1}$ and $Z_{2}$ represent the equivalent impedance on the transmitting side and receiving side, respectively, and $M$ represents the mutual inductance between the transmission and receiving coils which can be calculated by $M=\frac{N_{1} N_{2} \mu_{0}}{4 \pi} \oint_{C 1} \oint_{C 2} \frac{d l_{1} \cdot d l_{2}}{r_{12}}$ or electromagnetic simulation as described in $[20,21]$ for two paralleling solenoid coils. When transmitter and receiver are both in a resonant state, the transfer efficiency can be given by:

$$
\eta=\frac{(\omega M)^{2} R_{L}}{\left(r_{2}+R_{L}\right)\left[r_{1}\left(r_{2}+R_{L}\right)+(\omega M)^{2}\right]}
$$

Here $r_{1}, r_{2}$ represent the resistance of the transmitting and the receiving coil, $R_{\mathrm{L}}$ represents equivalent resistance of the online monitoring equipment from the receiving coil side. Mid-range wireless power transfer is taken into consideration since the insulation distance of a $110 \mathrm{kV} H V P L$ is $1.1 \mathrm{~m}$. When the distance between the transmitting and receiving coils is more than two times the coil diameter, the coupling coefficient experiences a cubic decay with the increase of transfer distance, leading to a drastic decline of the transfer efficiency. At this point, improving the transmission efficiency needs to enhance the reflected impedance of the wireless power transmission system, and the simplest way is to increase the resonant frequency [22]. If the insulation distance of $1.1 \mathrm{~m}$ is taken into account, the most appropriate frequency is set to $\mathrm{MHz}$ in the paper. In order to find out the efficiency feature of $110 \mathrm{kV} \mathrm{HVPL}$, simulation and computation are carried out by taking $R_{\mathrm{L}}$ into consideration. The power transmitting coils and receiving coils are spiral coils, which are tightly wound with Liz wire-1650/0.05F. Meantime, they are also designed with the same parameters, that is, the radius is $15 \mathrm{~cm}$ and the coil turn is 6. Results are shown in Figure 2.

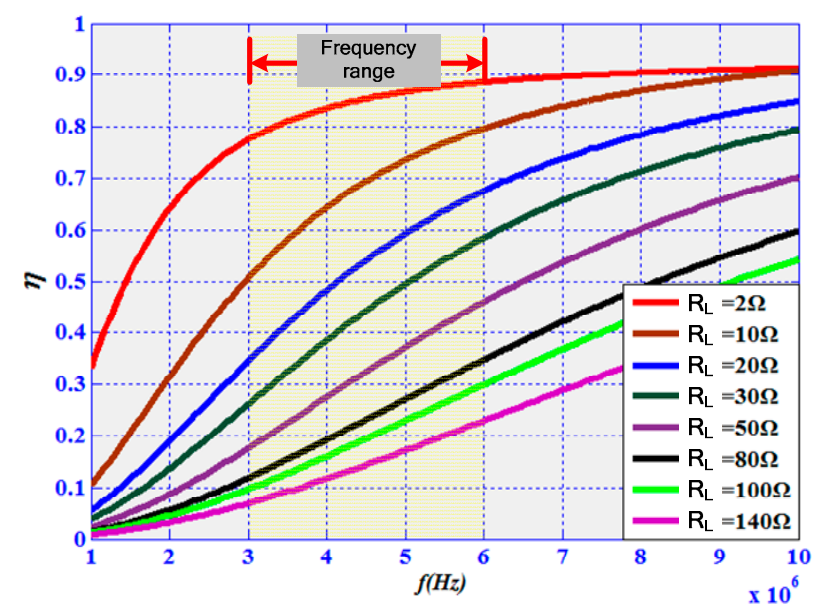

Figure 2. The efficiency varies with the resonant frequency under different resistance. 
It is obvious from Figure 2 that the higher the resonant frequency is, the higher the efficiency that could be achieved when the transmission distance is fixed. One thing that needs to be considered is that along with an extremely high frequency, the self-inductance of the coil is largely affected by external changes, making the system rather sensitive and may even cause drastic decrease of efficiency because of small deviations from the resonant frequency. To achieve both high efficiency and stability, the HVPL wireless power transmitting system is suggested to work around 3-6 MHz. From Figure 2 we can also find that system efficiency increases while load resistance decreases. It can reach an efficiency of $10 \%$ when the resonant frequency is $3-4 \mathrm{MHz}$ and the load resistance is $140 \Omega$. Considering the power requirements of online monitoring equipment, the wireless power supply system can work normally only when the maximum extracted power of the energy harvesting device is $100 \mathrm{~W}$. Consequently, a series of design optimizations of the energy harvesting device are necessary.

\section{Modeling and Optimization Design of Energy Harvesting Device}

\subsection{Theoretical Analysis}

Energy harvesting device extracts energy from magnetic fields around the HVPL, which is illustrated in Figure 3 [23].

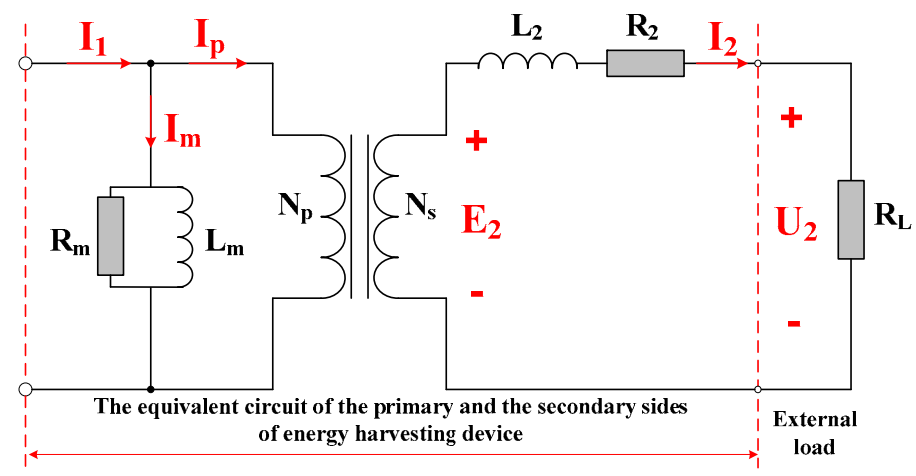

Figure 3. The equivalent circuit of the energy harvesting device.

Energy harvesting devices applied in HVPL are supposed to deliver stable power regardless of current fluctuations, because too large a current may lead to deep saturation of the core and cause serious overheating problems, so it is essential to make sure that the core works in linear areas, which is one of the design criteria. Through practical investigation, the current in $110 \mathrm{kV}$ HVPL in Yangzhou, Jiangsu Province, China is set to range from $20 \mathrm{~A}$ to $1000 \mathrm{~A}$. A gapped core is thus used to avoid core saturation and increase the maximum saturation magnetic flux density. Besides, it is of great convenience to install the energy harvesting device on the HVPL. According to Figure 3, the equivalent circuit equation is given by:

$$
\left\{\begin{array}{l}
I_{1}-I_{m}=I_{p} \\
I_{p} N_{p}=I_{2} N_{s} \\
E_{2}-U_{2}=I_{2}\left(R_{2}+j \omega L_{2}\right)
\end{array}\right.
$$

Here $I_{1}$ represents the current in the HVPL, $I_{\mathrm{m}}$ represents the excitation current. $N_{\mathrm{p}}$ and $N_{\mathrm{s}}$ represent the primary turn and the secondary one of the energy harvesting device, respectively. $I_{2}$ represents the secondary current. $E_{2}$ represents the induced voltage on the secondary side. $U_{2}$ represents the load voltage. $R_{2}$, and $L_{2}$ represent the resistance and inductance of the secondary coil. $R_{\mathrm{L}}$ represents equivalent resistance of the secondary side. To simplify the analysis, hysteresis loss, eddy current loss and other iron loss are ignored through the calculation. A gapped core structure is shown in Figure 4. 


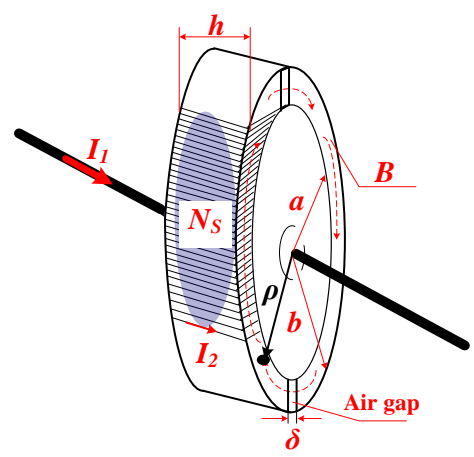

Figure 4. The structure of the energy harvesting device with an air gap.

It is assumed that the gap between two semicircular cores is $\delta$. According to the law of magnetic potential balance, it can be concluded that:

$$
\left\{\begin{array}{l}
N_{1} I_{m}=H_{e} l_{e}+H_{\delta} \cdot 2 \delta \\
N_{1} I_{m}=H^{\prime} l_{t}
\end{array}\right.
$$

Here $H_{\mathrm{e}}, l_{\mathrm{e}}$ represent the magnetic field strength and the magnetic length in the core, and $H^{\prime}, l_{\mathrm{t}}$ represent the overall magnetic field strength and the equivalent magnetic length in gapped core. Since $B=\mu H$, the equivalent permeability of the designed energy harvesting device can be given by:

$$
\mu^{\prime}=\frac{l_{t} \mu_{0} \mu_{r}}{\varepsilon l_{e}+\mu_{r} \cdot 2 \delta}
$$

Here $\varepsilon$ is the edge effect coefficient of the magnetic field near the gap, which in engineering applications, can be ignored under the condition of $\delta / h \leqslant 0.2$ and $\delta /(b-a) \leqslant 0.2$, when $\varepsilon=1$ [24]. According to Ampere's circuit theorem, the magnetic flux density at any radius $\rho$ can be obtained by:

$$
\boldsymbol{B}_{1}=\frac{\sqrt{2} \mu^{\prime} i_{m}(t)}{2 \pi \rho} \boldsymbol{a}_{\phi}
$$

The magnetic flux of the cross-linked ring core can be expressed by:

$$
\Phi_{m}(t)=\int_{s} B_{1} \cdot \mathrm{d} s=\frac{\sqrt{2} \mu^{\prime} i_{m}(t)}{2 \pi} \int_{a}^{b} \frac{1}{\rho} \mathrm{d} \rho \int_{0}^{h} \mathrm{~d} z=\frac{\sqrt{2} \mu^{\prime} I_{m} \cos (\omega t)}{2 \pi} \ln \left(\frac{b}{a}\right) h
$$

so the induced voltage on the secondary side can be calculated by:

$$
E_{2}=-N_{s} \frac{\mathrm{d} \Phi_{m}(t)}{\mathrm{d} t}=\sqrt{2} N_{s} \mu^{\prime} f I_{m} \sin (\omega t) \ln \left(\frac{b}{a}\right) h
$$

Here $f$ is the excitation current frequency. $a, b$ represent the inner and the outer radius of the core, respectively. When the device is working the power frequency, resistance $\left(R_{2}\right)$ and inductance $\left(L_{2}\right)$ of the secondary side can be calculated by [23]:

$$
\left\{\begin{array}{l}
L_{2}=\frac{\mu^{\prime} N_{S}}{2}(b-a) h \sqrt{\frac{\pi}{S_{c u}}} \cdot K \\
R_{2}=2(b-a+h) N_{s} \rho_{c u} / S_{c u}
\end{array}\right.
$$

Here $\rho_{c u}, S_{c u}$ denote the resistivity and the cross-sectional area of the copper wire. $\rho_{c u}=0.0175 \Omega \cdot \mathrm{mm}^{2} / \mathrm{m}$ when temperature is $20^{\circ} \mathrm{C}$. $\mathrm{K}$ denotes the effective coefficient of the rectangle cross-sectional coil, which is determined by the ratio of the height of the core $h$ and the length of the 
secondary coil. When Equations (9) and (10) are integrated, the expression of the secondary current is obtained and expressed by:

$$
\left\{\begin{array}{l}
I_{2}(t)=\frac{2 \sqrt{2} N_{s} \mu^{\prime} f I_{m} \sin (\omega t) \ln \left(\frac{b}{a}\right) h \cdot S_{c u}}{j \omega \mu^{\prime} N_{s}(b-a) h \sqrt{\pi S_{c u}} \cdot K+4(b-a+h) N_{s} \rho_{c u}+2 R_{L} \cdot S_{c u}} \\
I_{m}=I_{1}-N_{s} I_{2}
\end{array}\right.
$$

Assuming: $a_{1}=2 \sqrt{2} N_{s} \mu^{\prime} f \ln (b / a) h \cdot S_{c u}, a_{2}=\omega \mu^{\prime} N_{s}(b-a) h \sqrt{\pi S_{c u}} \cdot K, a_{3}=4(b-a+h) N_{s} \rho_{c u}+$ $2 R_{L} \cdot S_{c u}$, the real and imaginary part of secondary current can be expressed by: scale and align

$$
\left\{\begin{array}{l}
\operatorname{Re}\left(I_{2}\right)=\frac{a_{1} a_{3}\left(a_{2}^{2}+a_{3}^{2}+N_{s} a_{1} a_{3}\right)+N_{s} a_{1}^{2} a_{2}^{2}}{\left(a_{2}^{2}+a_{3}^{2}+N_{s} a_{1} a_{3}\right)^{2}+N_{s}^{2} a_{1}^{2} a_{2}^{2}} \cdot I_{1} \\
\operatorname{Im}\left(I_{2}\right)=\frac{N_{s} a_{1}^{2} a_{2} a_{3}-a_{1} a_{2}\left(a_{2}^{2}+a_{3}^{2}+N_{s} a_{1} a_{3}\right)}{\left(a_{2}^{2}+a_{3}^{2}+N_{s} a_{1} a_{3}\right)^{2}+N_{s}^{2} a_{1}^{2} a_{2}^{2}} \cdot I_{1}
\end{array}\right.
$$

It is obvious that primary current is not completely linearly proportional to the secondary current because of the nonlinear coefficient $K$ in Equation (11) caused by the excitation current. The receiving power of the load can be further calculated by:

$$
P_{o}=\left[\left(\operatorname{Re}\left(I_{2}\right)\right)^{2}+\left(\operatorname{Im}\left(I_{2}\right)\right)^{2}\right] \cdot R_{L}
$$

\subsection{Parameters Optimization Design with Threshold Constraints}

\subsubsection{Parameter Optimization Design of the Core}

When the current is small, it is necessary to increase the magnetic induction intensity B so that enough power can be obtained. Considering that the gap length $\delta$ has a significant influence on the equivalent permeability, a short gap length (normally less than $1 \mathrm{~mm}$ ) is preferable in this circumstance. In accordance to Equations (5) and (6), the magnetic induction intensity constraints can be given by:

$$
\left\{\begin{array}{l}
B_{r} \leqslant \frac{I_{m \min } \cdot \mu^{\prime}}{l_{t}} \\
B_{m \max } \geqslant \frac{I_{m \max } \cdot \mu^{\prime}}{l_{t}}
\end{array}\right.
$$

Equation (14) demonstrates that core materials exert a direct effect on the electricity extracted along with current fluctuations. On the one hand, a high initial permeability is needed to achieve enough output power when the current is small. On the other hand, a high saturation magnetic flux density coefficient is necessary to avoid premature saturation when the current is large. Through comparison among features of different materials, cold rolled silicon steel sheet is selected for the core. Specifically, the initial permeability of this material reaches $1000 \mathrm{H} / \mathrm{m}$ and the saturation magnetic flux density reaches $2.03 \mathrm{~T}$.

While the device is working at the power frequency, the suppression effect of the secondary inductance on the current is small. Moreover, the self-resistance of the secondary coil is insensitive to the core size. As a result, the secondary coil is assumed to have no influence on output power. The secondary current reaches its maximum when the secondary induced voltage $E_{2}$ is maximized. According to Equations (8) and (9), the relationship between induced voltage $E_{2}$ and core parameters can be expressed by:

$$
E_{2} \propto\left\{\begin{array}{l}
\Phi_{m}\left(\ln \frac{b}{a}, h\right) \Rightarrow \Phi_{m}\left(S_{e q}\right) \\
\mu^{\prime}(a)
\end{array}\right.
$$


It is observed from equation (15) that two design principles should be followed: (1) large inner radius $a$; (2) large cross-sectional $S_{e q}$. If core height $h$ and core width $(b-a)$ are both adjustable, core width $(b-a)$ will have a greater influence on output power because of the $K$ changes caused by $(b-a)$. However, the core width must satisfy $\delta /(b-a)<0.2$ to avoid the edge effect of magnetic fields, making the selection of core inner radius $a$ much more difficult. Furthermore, it is impossible to extend $S_{e q}$ in engineering applications for the reason that excessive weight of the core may result in wire deformation. On the contrary, it is essential to decrease $S_{e q}$ as much as possible on the premise of sufficient output power.

Compared with core size, gap $\delta$ has a greater influence on the initial current, core saturation as well as the extracted electricity. A gapped core can generate a larger magnetic field intensity when the magnetic induction intensity is the same, which means the gap helps to broaden the linear working region. As a consequence, output power should be guaranteed to be large enough when the primary current is small and the core is not oversaturated when the secondary circuit is open, so the gap design should satisfy the following equation:

$$
\left\{\begin{array}{l}
\pi a \cdot \frac{\mu_{0} \mu_{r} I_{1 \max }-\gamma B_{\max }}{\mu_{r}\left(\gamma B_{\max }-\mu_{0} I_{1 \max }\right)}<\delta \leqslant \pi b \cdot \frac{\mu_{0} \mu_{r} N_{2} f I_{m \min } \ln \left(\frac{b}{a}\right) h-E_{2 \min }}{\mu_{r}-\mu_{0} \mu_{r} N_{s} f I_{m \min } \ln \left(\frac{b}{a}\right) h} \\
\delta<0.2(b-a) \cap \delta<0.2 h
\end{array}\right.
$$

Here $I_{1 \max }$ is the maximum primary current, $I_{\min }$ is the minimum excitation current. $E_{2 \min }$ is the minimum induced voltage needed by the load. $\gamma$ is the saturation coefficient, which should be set to guarantee that both numerator and denominator on the left side of the inequality are positive. Similarly, the value of $\delta$ should ensure that both numerator and denominator on the right side of the inequality are positive.

\subsubsection{Design Optimization of the Secondary Coil}

Compared with the core parameters which mainly affect system stability, the turns of the secondary coil $N_{S}$ and the load $R_{L}$ have a larger influence on the output power, which is more sensitive to $N_{s}$. When the primary current is small, larger $N_{s}$ can increase the output power and decrease the initiating current of the energy harvesting device. On the contrary, a smaller $N_{S}$ is preferable when the primary current is large. Taking all the factors above into consideration, the design of secondary coil should meet the following rules:

(1) The inner resistance of the coil should not be larger than the load resistance to ensure maximum output power:

$$
N_{s} \leqslant \frac{R_{L} S_{c u}}{2(b-a+h) \rho_{c u}}
$$

(2) Since the core is composed of two semicircles, a maximum $N_{s}$ is achieved when the secondary coil covers half of the core at most, which means $N_{s}$ is determined by the inner radius of the core:

$$
N_{s} \leqslant \frac{\pi a}{2} \sqrt{\frac{\pi}{S_{c u}}}
$$

\section{Results}

\subsection{Simulation Analysis of the Energy Harvesting Device}

In this part, the optimal parameters of the power harvesting device are determined with threshold constraints to demonstrate the direct influences of those parameters on output power. Simulation is carried out to visually display the calculations and the discussions in Section 3. Though the specific working environment is determined in advance, any major conclusions drawn from the analysis should 
be suitable for other circumstances too. An example of the practical design of a power harvesting device is presented here.

The current in the HVPL is assumed to be $500 \mathrm{~A}$. Cold rolled silicon steel sheet is selected for the core according to 3.2.1. Further analysis of parameter optimization factors such as core inner radius $a$, core external radius $b$, radial thickness $(b-a)$, height $h$, gap $\delta$ and coil turns $N_{s}$ is introduced to find out the general rules for achieving maximum output power.

Since multiple parameters interact with each other, the dimensions of the analysis are reduced step by step to simplify the analysis and ensure the accuracy at the same time. The analytical method adopted in the paper is fractional reduction of dimensions, that is, the influences of a parameter with less impact on the output power is analyzed first, and then a certain law can be obtained. If this complies with the principle of monotony, this parameter can be set as a reference point when we analyze how other parameters affect output power. If not, then this parameter may interact with others, so a second parameter should be chosen.

After a series of computational analysis and dimension reduction analyses, core inner radius $a$, radial thickness $(b-a)$ are analyzed first. The results are shown in Figure 5.

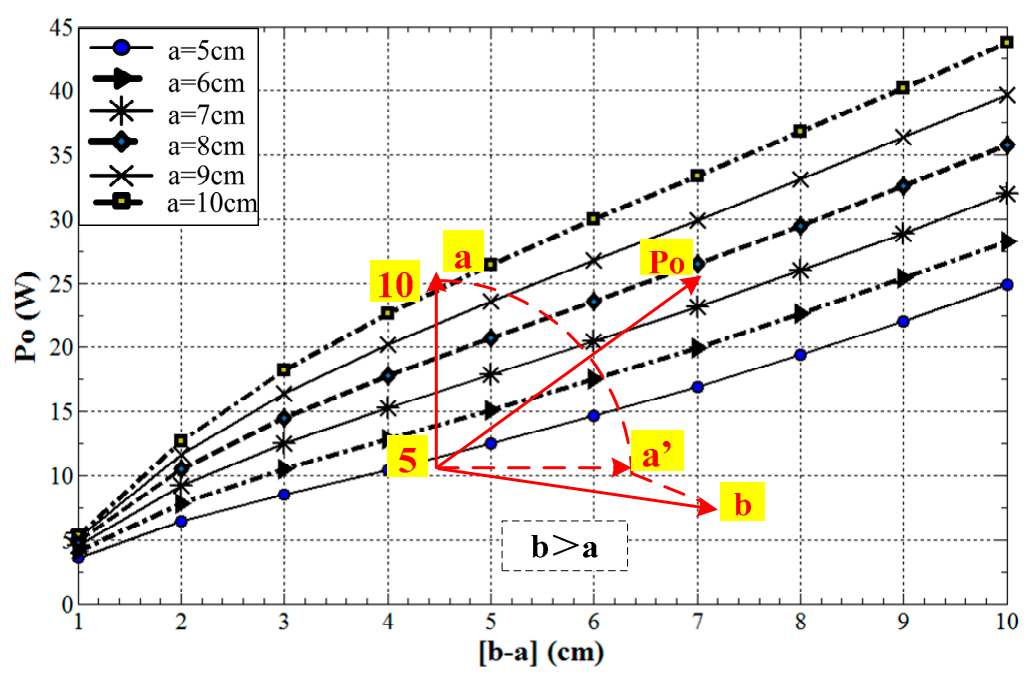

Figure 5. The relationship between the harvested energy and the inner/external diameter of the core.

It is apparent from Figure 5 that output power increases as the radial thickness increases $(b-a)$. Besides, a larger core inner radius contributes to more output power when other parameters remain the same, which corresponds with the theoretical analysis. It is surprising to find that $40 \mathrm{~W}$ is obtained without any other optimization. Since both the core inner radius and radial thickness have a monotonic increasing relationship with output power, which meets the criteria of dimension reduction analysis, parameters $a$ and $(b-a)$ are determined first. Additionally, too large a core inner radius $a$ and radial thickness $(b-a)$ have much smaller influences on the increase of output power and may increase the risk of wire deformation. Thus, suitable evaluation parameters should be chosen in advance to do further optimization.

To verify the specific influence of core cross-sectional area $S_{e q}$ on output power illustrated in Equation (15), radial thickness $(b-a)$ and core height $h$ are chosen to assess their impacts on determining the output power. Similarly, the changing trend while other parameters remain the same is shown in Figure 6. 


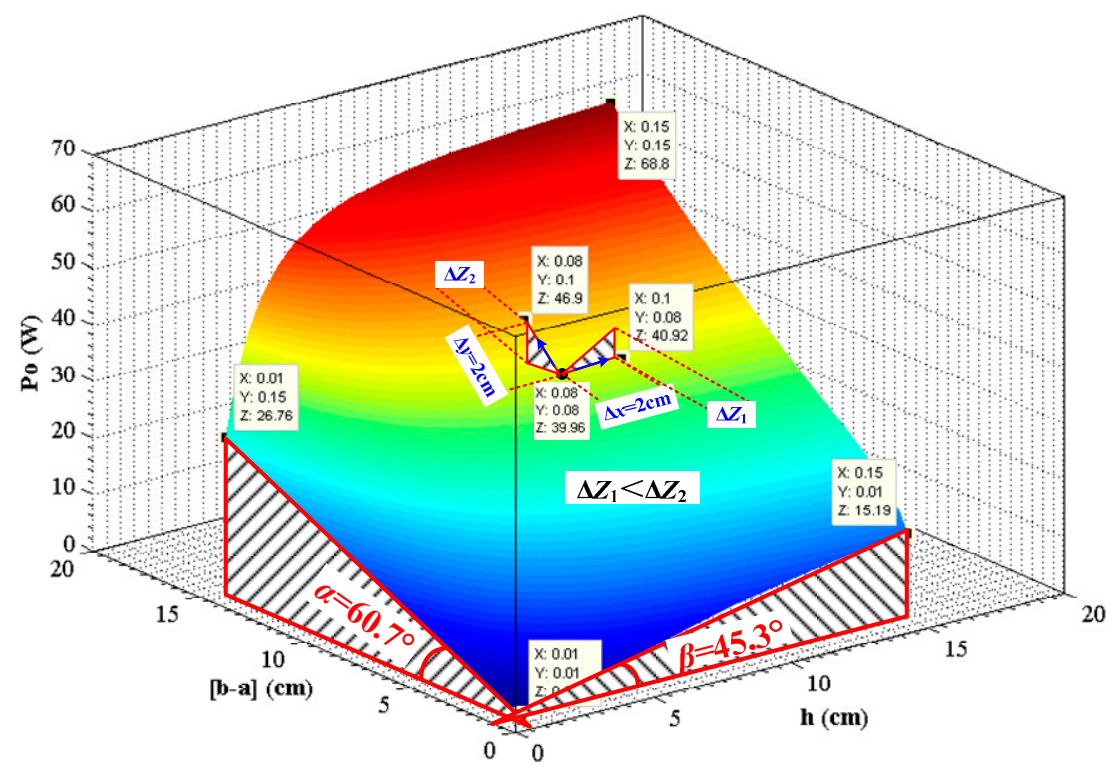

Figure 6. The relationship between the harvested energy and the thickness/axial height of the core.

As shown in Figure 6, output power increases with the increase of $S_{e q}$, and the rise of the radial thickness $(b-a)$ and core height also leads to the increase of output power. An important distinction between these two dimensions, namely, $(b-a)$ and $h$ is that same increase leads to different output power changes. Take $\left\{\begin{array}{l}{[b-a]=h=8 \mathrm{~cm}} \\ P_{o}=39.96 \mathrm{~W}\end{array}\right.$ as a reference point, $\Delta Z_{1}=\Delta P_{\mathrm{o} 1}=0.96 \mathrm{~W}$ when $\Delta x=\Delta h=2 \mathrm{~cm}$ but $Z_{2}=\Delta P_{\mathrm{o} 2}=6.94 \mathrm{~W}$ when $\Delta y=\Delta h=2 \mathrm{~cm}$. Apparently, $\Delta Z_{2}$ is $5.98 \mathrm{~W}$ more than $\Delta Z_{1}$ which is in accordance with theoretical analysis.

In sum, increasing the core inner radius and cross-sectional areas both lead to larger output power. However, the increase rate in Figures 5 and 6 is pretty small, so these optimization methods commonly serve as helping measures. As a result, optimizations of the core gap $\delta$ and secondary coil turns $N_{\mathrm{s}}$ are discussed to increase the output power by a large margin. A part of parameters are determined as follows: core inner radius $a=10 \mathrm{~cm}$, core external radius $b=18 \mathrm{~cm}$, core height $h=8 \mathrm{~cm}$. The relationship between output power and core gap as well as secondary coil turns is displayed in Figure 7.

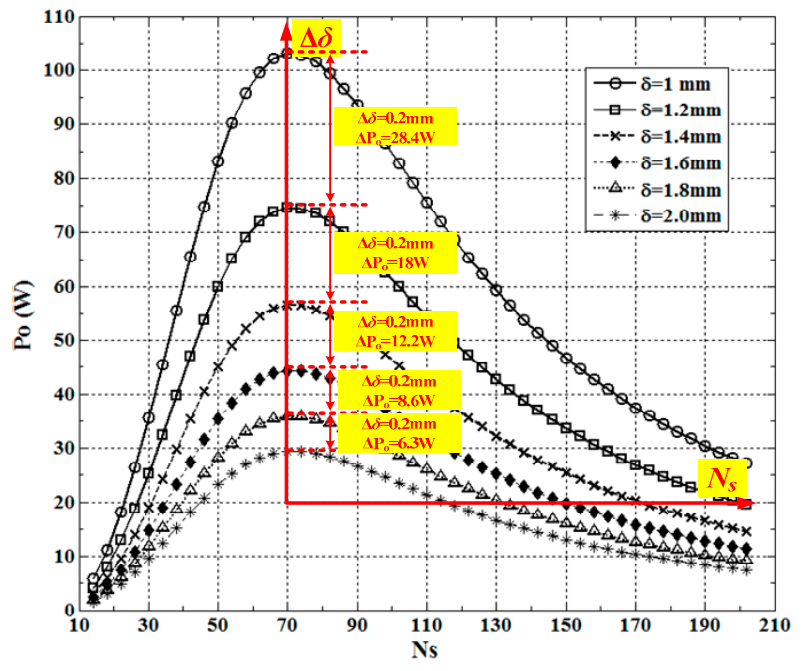

Figure 7. The harvested energy varies with the air gap and the turns of secondary windings. 
It is obvious from Figure 7 that there is an optimal secondary coil turn where output power is maximized. Meanwhile, output power increases prominently with the decrease of core gap $\delta$. For instance, when $\delta$ decreases from $2 \mathrm{~mm}$ to $1.8 \mathrm{~mm}$, the maximum output power increases by $6.3 \mathrm{~W}$. When $\delta$ decreases from $1.2 \mathrm{~mm}$ to $1 \mathrm{~mm}$, the maximum output power increases by $28.4 \mathrm{~W}$. In conclusion, for a core of fixed parameters, maximum output power can only be achieved with a certain secondary coil turn. When the primary current is $500 \mathrm{~A}$, the optimum core dimension is suggested as follows: core inner radius $a=10 \mathrm{~cm}$, core external radius $b=18 \mathrm{~cm}$, core height $h=8 \mathrm{~cm}$, core gap $\delta=1 \mathrm{~mm}$ and secondary coil turns $N_{\mathrm{s}}=60-80$.

Though a smaller core gap contributes to larger output power, it is impossible to minimize the core gap as much as possible since the core gap plays an important role in broadening the linear working regions of the core. In a situation when the gap is small and the current is large, the core is likely to work in deep saturation mode which will result in a drastic rise in temperature. Therefore it is very necessary to keep the energy extracting device working in the critical range between linear and saturated regions. In order to increase the output power when the current is small and discharge output power when current is large, a stability control strategy of extracting energy is proposed to adjust to the fluctuation of current. The basic principle is to control the core gap and the secondary coil turn as shown in Figure 7. An overall scheme of the control strategy is specifically illustrated in Figure 8.

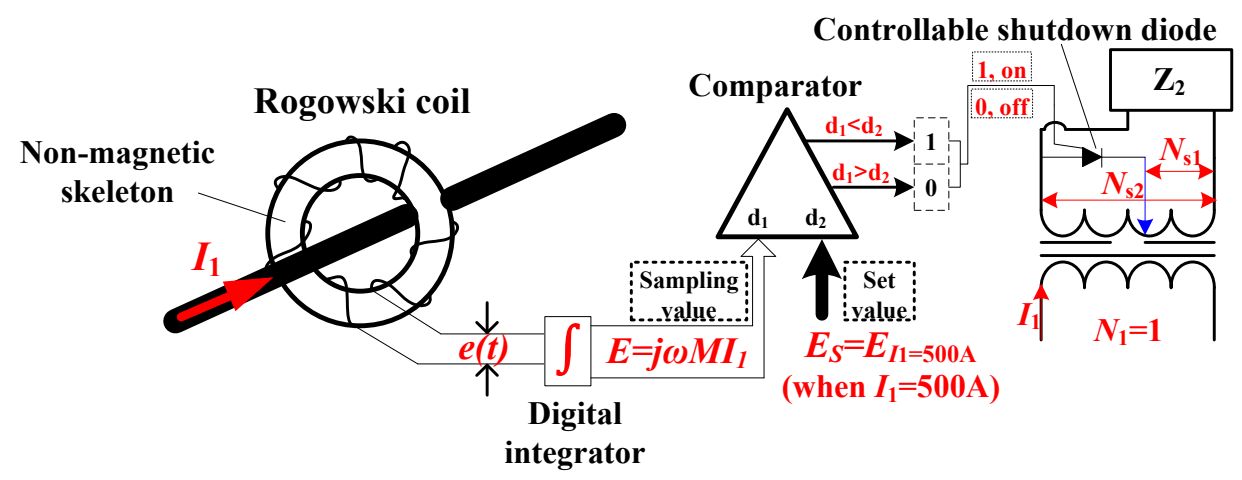

Figure 8. The control strategy of power stability.

In Figure 8, the control strategy is divided into the following steps:

(1) According to Figure 7, both the optimal secondary coil turn $N_{\mathrm{s} 1}$ to achieve maximum output power and secondary coil turn $N_{\mathrm{s} 2}$ to ensure the minimum demanded power can be determined, which should comply with $N_{\mathrm{s} 2}>N_{\mathrm{s} 1}$.

(2) Use a Rogowski coil to sample the current of HVPL in the form of induced voltage. A digital integrator is applied to transform the transient voltage signal $e(t)$ into a stable signal $E$. The sampled voltage when the current equal to $500 \mathrm{~A}$ (less than or equal to the saturation current of core) is set as the standard value.

(3) The digital comparator is in charge of comparing the sampled $E$ with the standard value. It outputs high-level signals (" 1 ") when $\mathrm{d} 1<\mathrm{d} 2$ and outputs low-level signals (" 0 ") when $\mathrm{d} 1>\mathrm{d} 2$.

(4) The diode is shut off when the comparator outputs high-level signals and the secondary coil turn is set to be $N_{\mathrm{s} 1}$ so that maximum output power is achieved. On the contrary, the diode is turned on and the secondary coil turn is set to be $N_{\mathrm{s} 2}$ so that minimum demanded power is acquired. The control strategy greatly reduces the impact of power increase on the load and realizes the goal to obtain relatively stable output power, regardless of current fluctuation.

If the comparator shown in Figure 8 is equipped with multiple channels, several preset values can be set accordingly, so that the turns of secondary coil will be divided into several segments by 
controllable diodes. This can bring about two benefits: (1) the receiving power can be regulated to be more stable when the HVPL current is in fluctuation; (2) in addition, the core saturation can be limited by changing the turns of secondary coil to increase secondary current when HVPL current is too large. What we need to do is just to determine the most suitable number of turns of the secondary coil.

\subsection{Experimental verification}

To verify the accuracy of theoretical and simulated analysis, multiple paired experiments are carried out by using the designed devices as shown in Figure 9. A single wire is enwound into several turns to generate large primary current so that a real working status of HVPL can be simulated. An iodine-tungsten lamp $(220 \mathrm{~V}, 1000 \mathrm{~W})$ is connected to the source $(220 \mathrm{~V}, 50 \mathrm{~Hz})$ with a wire to generate the primary current in the experiments. When the current in single wire is $4.1 \mathrm{~A}$ and wire turns is 25 , then the primary current of power harvesting device is $102.5 \mathrm{~A}$.

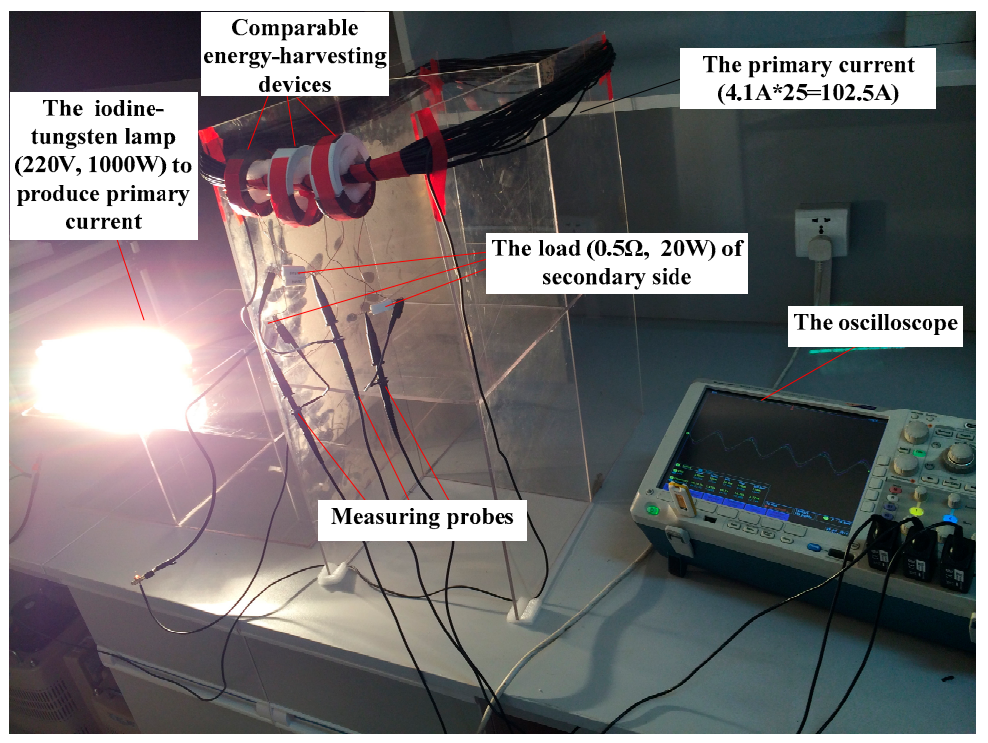

Figure 9. Experimental system for optimal design of the energy harvesting device.

With regard to the parameters optimization design with threshold constraints, nine paired experiments are designed to implement comprehensive validations, when the condition of $\delta / h \leqslant 0.2$ and $\delta /(b-a) \leqslant 0.2$ is considered. Specific experimental parameters are shown in Table 1.

Table 1. Comparative experimental parameters of nine energy harvesting devices $\left(I_{1}=102.5 \mathrm{~A}\right.$, $R_{L}=0.5 \Omega$ ).

\begin{tabular}{ccccccc}
\hline Device Number & $\boldsymbol{a}(\mathbf{c m})$ & $(\boldsymbol{b}-\boldsymbol{a}) \mathbf{( c m})$ & $\boldsymbol{h}(\mathbf{c m})$ & $\boldsymbol{\delta}(\mathbf{m m})$ & $\boldsymbol{N}_{\mathbf{s}}$ & $\boldsymbol{P}_{\boldsymbol{L}}(\mathbf{W})$ \\
\hline 1 & 2.25 & 1.0 & 1.65 & 1 & 100 & 2.25 \\
2 & 2.75 & 1.0 & 1.65 & 1 & 100 & 4.84 \\
3 & 2.25 & 1.1 & 1.65 & 1 & 100 & 6.25 \\
4 & 2.25 & 1.0 & 1.75 & 1 & 100 & 4.00 \\
5 & 2.75 & 1.0 & 1.65 & 1 & 30 & 1.69 \\
6 & 2.75 & 1.0 & 1.65 & 1 & 50 & 3.24 \\
7 & 2.75 & 1.0 & 1.65 & 1 & 80 & 5.76 \\
8 & 2.75 & 1.0 & 1.65 & 0.5 & 100 & 17.64 \\
9 & 2.75 & 1.0 & 1.65 & 2 & 100 & 1.96 \\
\hline
\end{tabular}

Here, $a,(b-a), h, \delta$, and $N_{\mathrm{s}}$ are in short for the inner radius, the radial thickness, the core height, the air gap, and the secondary coil turns of the core, respectively. Similarly, the output power of the energy harvesting device is represented as $P_{L}$. 
Test 1: the influence of $0.5 \mathrm{~cm}$ 's increase in core inner radius on output power is studied by comparing 1 and 2 in Table 1.

Test 2: 1, 3 and 4 are analyzed comparatively to investigate the influence of core radial thickness (b-a) and core height $h$ on output power when the two parameters are increased by $0.1 \mathrm{~cm}$, respectively.

Test 3: the influence of core gap $\delta$ on output power is demonstrated through a comparison among 2,8 and 9 .

Test 4: 2, 5, 6 and 7 are tested to verify the influence of secondary turns $N_{S}$ on output power.

The corresponding results of Test 1-Test 4 are displayed in Figure 10a-d. Several conclusions can be drawn from the experimental results above:

(1) Output power increases with the increase of core inner radius when other parameters remain certain;

(2) The increase of core radial thickness $(b-a)$ and core height $h$ will contribute to the increase of output power but the former one has a more pronounced effect;

(3) The smaller core gap is, the more output power the device can obtain once it holds true for Equation (16) and other parameters remain unchanged;

(4) Maximum output power can be acquired with the optimized secondary coil turns when other parameters remain certain. In this experiment specifically, $P_{o}\left(N_{s}=80\right)>P_{o}\left(N_{s}=100\right)$.

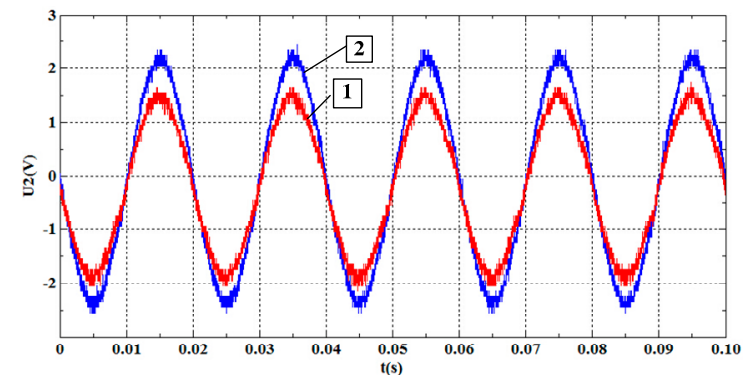

(a)

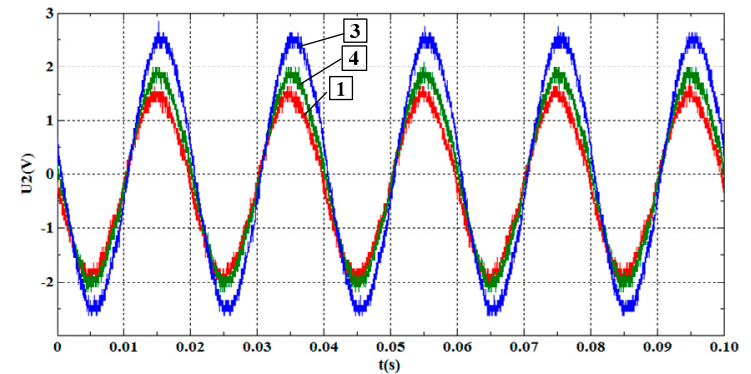

(b)

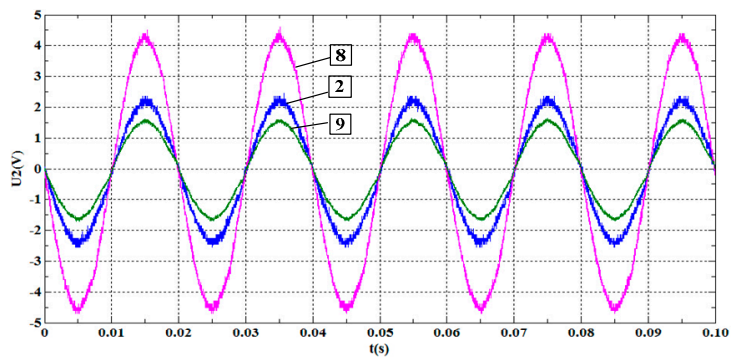

(c)

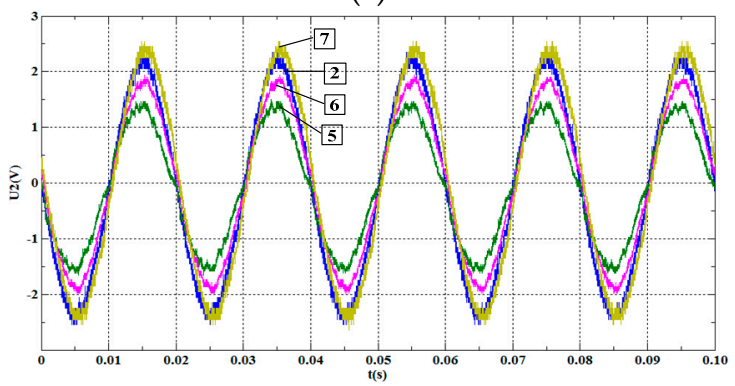

(d)

Figure 10. Experimental results: (a) Load voltage contrast between 1 and 2 of the energy harvesting device; (b) Load voltage contrast between 1, 3 and 4 of the energy harvesting device; (c) Load voltage contrast between 2, 8 and 9 of the energy harvesting device; (d) Load voltage contrast between 2, 5, 6 and 7 of the energy harvesting device.

In order to further validate the proposed optimization theory, the power calculations are performed when the parameters remain the same as that of experiments, which are shown in Table 1. The theoretical calculated results are compared with those of the experiments, as shown in Figure 11. 


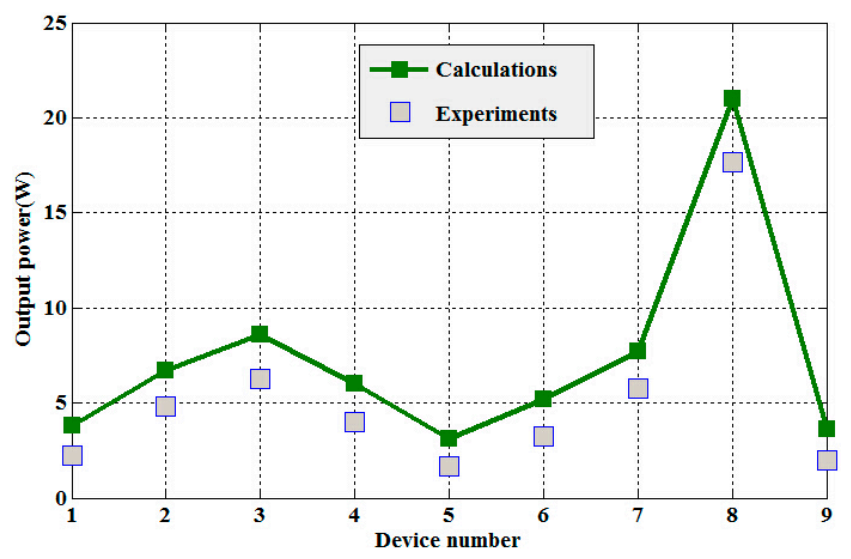

Figure 11. Calculations and experiments of output power under different energy harvesting devices.

The primary calculated current is the same as that seen in the experiments, that is, $102.5 \mathrm{~A}$. From Figure 11, it is obvious that the calculated load power and experimental results have the same trend, which proves the accuracy of the theoretical analysis. There are some misalignments between calculations and experiments that are mainly caused by the different formations of the primary current. In the experiments, the primary current is generated by 25 turns of wires, and the current in a single wire is $4.1 \mathrm{~A}$. For the small radius of every wire, the wires in the middle of the group would have little contribution to the main flux of the core, so the effect of the equivalent primary current in the experiments is actually less than that of the single thick wire with the same current value, which is the main reason why the extracted power in the experiments is less than that in the calculations.

\section{Discussion}

Due to the large potential difference between the power line and a transmission tower, it is difficult to safely transfer enough energy to monitoring devices. The emergence of wireless power transfer technology provides an alternative way to solve the insulation problem since it does not need any power cables. The technology has been widely applied in fields such as charging of electric vehicles, home appliances and implantable medical devices, but never in powering HVPL monitoring devices. What we need to tackle is to how provide enough energy for the transmission side with an inductive energy harvesting device, which is also the main concern in this paper. Though many inductive energy harvesting devices have already been developed, their design methodology is based on the traditional $\mathrm{CT}$ for measurements and experimental tests. As a result, the experimental prototypes cannot provide enough power or solve the problem of voltage distortion. As far as we are concerned, there is no previous research relevant to the design of high-power inductive energy harvesting devices. In the paper, an ptimization design of inductive energy harvesting device is introduced, which is also suitable for other HVPL configurations. Then the extracted energy can be transformed into high-frequency alternating current power to power the transmission coil, which is our future research focus. Chances are high that the proposed combination of inductive energy harvesting and a wireless power supply system could be used in engineering applications. This will undoubtedly bring about a breakthrough in the field of energy conversion and transmission in the power system.

\section{Conclusions}

The solutions to power $110 \mathrm{kV}$ HVPL online monitoring equipment on the tower are investigated which in the paper are based on the supply demand of the monitoring equipment and the transfer efficiency of the wireless power transmission system. Firstly, an online power harvesting device is modeled and specific parameters affecting the extracted energy are analyzed. The initiating current and saturation features are also taken into consideration to determine the threshold constraints of 
device parameters. Secondly, an optimization design of the power harvesting device is carried out to increase the extracted energy. Thirdly, experimental verifications are conducted to demonstrate the feasibility of the proposed optimization methods. It is observed that a larger core inner radius, core radial thickness, core height and smaller gap air can increase the extracted energy but exert different impacts. Moreover, the maximum extracted power can be achieved when the secondary coil turns are optimized. Finally, a power stabilization strategy is put forward so that more power can be extracted when the current is small and extra energy will be discharged when the current is large. The work of this paper is of great importance to the design of high-power magnetic field energy harvesting for wireless power supply systems in overhead high-voltage power lines.

Acknowledgments: This work was supported in part by The Fundamental Research Funds for the Central Universities, Research Innovation Program for College Graduates of Jiangsu Province (No. KYLX15_0133), National Nature Science Youth Foundation of China (No. 51507032) and Nature Science Youth Foundation of Jiangsu Province (No. BK20150617).

Author Contributions: Wei Wang designed the model and performed the calculation and experiments. Wei Wang, Xueliang Huang and Linlin Tan proposed the research topic and analyzed the data. Jinpeng Guo and Han Liu helped write a part of programs in simulation. All authors contributed to the writing of the manuscript, and have read and approved the final manuscript.

Conflicts of Interest: The authors declare no conflict of interest.

\section{References}

1. Lu, S.; Repo, D.G.; Della, F.A.; Figuerola, A.L.; Pikkarainen, M. Real-time low voltage network monitoring-ICT architecture and field test experience. IEEE Trans. Smart Grid 2015, 6, 2002-2012. [CrossRef]

2. Bobowski, J.S.; Ferdous, M.S.; Johnson, T.; Pikkarainen, M. Calibrated single-contact voltage sensor for high-voltage monitoring applications. IEEE Trans. Instrum. Meas. 2015, 64, 923-934. [CrossRef]

3. Xia, J.; Jiale, S.; Deng, X.; Wang, L.; He, S.; Liu, K. Enhanced transmission line pilot impedance and pilot protection. IET Gener. Transm. Distrib. 2011, 5, 1240-1249. [CrossRef]

4. Moser, M.J.; Bretterklieber, T.; Zangl, H.; Brasseur, G. Strong and weak electric field interfering: Capacitive icing detection and capacitive energy harvesting on a 220-kV high-voltage overhead power line. IEEE Trans. Ind. Electron. 2011, 58, 2597-2604. [CrossRef]

5. Han, J.C.; Hu, J.; Yang, Y.; Wang, Z.X.; Wang, S.X.; He, J.L. A nonintrusive power supply design for self-powered sensor networks in the smart grid by scavenging energy from AC power line. IEEE Trans. Ind. Electron. 2015, 62, 4398-4407. [CrossRef]

6. Moser, M.J.; Zangl, H.; Bretterklieber, T. An autonomous sensor system for monitoring of high voltage overhead power supply lines. Elektrotech. Informationstech. 2009, 126, 214-219. [CrossRef]

7. Zhao, X.; Keutel, T.; Baldauf, M. Energy harvesting for a wireless-monitoring system of overhead high-voltage power lines. IET Gener. Transmiss. Distrib. 2013, 7, 101-107. [CrossRef]

8. Wu, Z.; Wen, Y.; Li, P. A Power Supply of Self-Powered Online Monitoring Systems for Power Cords. IEEE Trans. Energy Convers. 2013, 28, 921-928. [CrossRef]

9. Dai, Y.; Chen, K.; Lin, D.; Cheng, Y.C.; Nie, M.; Wang, W. Design of induced power apparatus for monitoring system of dynamic increasing capacity of high-voltage transmission lines. In Proceedings of the 2012 International Conference on Condition Monitoring and Diagnosis, Bali, Indonesia, 23-27 September 2012.

10. He, Z.; Nie, S.; Qu, G.; Liu, Y.; Sheng, G.; Jiang, X. The design of CT energy harvesting power supply based on phase-controlled method. In Proceedings of the 2012 Asia-Pacific Power and Energy Engineering Conference, Shanghai, China, 1-5 March 2012.

11. Yao, M.; Zhao, M. Research on electric energy harvesting from high-voltage transmission line. In Proceedings of the 2013 3rd International Conference on Electric Power and Energy Conversion Systems, Istanbul, Turkey, 2-4 October 2013.

12. Banting, J.F.; Mcbee, B.W. Power Line Energy Harvesting Power Supply. U.S. Patent 20100084920 B2, 26 November 2013. Available online: https://patentscope.wipo.int/search/zh/detail.jsf?docId=US $43743066 \&$ recNum $=1 \&$ maxRec $=\&$ office $=\&$ prevFilter $=\&$ sortOption $=\&$ queryString $=\&$ tab $=$ NationalBiblio (accessed on 26 November 2013). 
13. Yuan, S.; Huang, Y.; Zhou, J.F.; Xu, Q.; Song, C.Y.; Thompson, P. Magnetic field energy harvesting under overhead power lines. IEEE Trans. Power Electron. 2015, 30, 6191-6202. [CrossRef]

14. Van Schalkwyk, J.A.; Hancke, G.P. Energy harvesting for wireless sensors from electromagnetic fields around overhead power lines. In Proceedings of the 2012 IEEE International Symposium on Industrial Electronics, Hangzhou, China, 28-31 May 2012.

15. Zhang, D. Potential Power Supply and Signal Transmission System of Online Monitoring Device on High Voltage Transmission Line. CN Patent 201210224012.3, 22 January 2014.

16. Kurs, A.; Karalis, A.; Moffatt, R.; Joannopoulos, J.D.; Fisher, P.; Soljacic, M. Wireless power transfer via strongly coupled magnetic resonance. Science 2007, 317, 83-86. [CrossRef] [PubMed]

17. Li, P.; Wen, Y.; Zhang, Z.; Pan, S. A high-efficiency management circuit using multi-winding up-conversion current transformer for power-line energy harvesting. IEEE Trans. Ind. Electron. 2015, 62, 6327-6335. [CrossRef]

18. Zhang, W.; Wong, S.C.; Tse, C.K.; Cheng, Q.H. Analysis and comparison of secondary series-and parallel-compensated inductive power transfer systems operating for optimal efficiency and load-independent voltage-transfer ratio. IEEE Trans. Power Electron. 2014, 29, 2979-2990. [CrossRef]

19. Moorey, C.; Holderbaum, W.; Potter, B. Investigation of high-efficiency wireless power transfer criteria of resonantly-coupled loops and dipoles through analysis of the figure of merit. Energies 2015, 8, 11342-11362. [CrossRef]

20. Zhang, Z.; Chau, K.T.; Liu, C.H.; Li, F.H.; Ching, T.W. Quantitative Analysis of Mutual Inductance for Optimal Wireless Power Transfer via Magnetic Resonant Coupling. IEEE Trans. Magn. 2014, 50, 1-4. [CrossRef]

21. Huang, X.L.; Wang, W.; Tan, L.L.; Zhao, J.M.; Zhou, Y.L. Study of transmission performance on strong coupling wireless power transfer system in free position. In Proceedings of the PIERS 2012 Electromagnetics Research Symposium, Moscow, Russia, 23 August 2012.

22. Duong, T.P.; Lee, J.W. A dynamically adaptable impedance-matching system for midrange wireless power transfer with misalignment. Energies 2015, 8, 7593-7617. [CrossRef]

23. Houtzager, E.; Rietveld, G.; Dessens, J.; Heemskerk, M.; van den Brom, H.E. Design of a non-invasive current sensor for application on high voltage lines. In Proceedings of the 2014 IEEE Conference on Precision Electromagnetic Measurements, Rio de Janeiro, Brazil, 24-29 August 2014.

24. Chen, T.M.; Liu, B.A.; Luo, Y.L. The Inductance of Coils with Special Shape and Special Purpose, 3rd ed.; Machinery Industry Press: Beijing, China, 1992; pp. 373-416. 